\title{
Making the Invisible Visible: Virtual/ Interactive Itineraries in Roman Padua
}

\author{
Maurizio Perticarini \\ Chiara Callegaro
}

Abstract

PD-Invisible aims to enhance the archaeological heritage of Padua hidden by urban development. The focus of the process is the creation of an adaptive AR App according to the type of user: professionals and researchers on the one hand and cultural tourism on the other. The workflow identifies a path through the city and connects the artifacts studied by the research; catalogue historical and archival documents; detects the structures through laser scanning and photogrammetry technologies; optimizes the acquired models both from a graphic point of view and through Scan to BIM; develops the AR App in Unity 3D. The research offers further insights assuming the use of artificial intelligence for this type of applications: differentiate the contents of the App according to the user's preferences by comparing the GPS data with those of the detection devices (camera and Lidar).

Keywords

augmented reality, BIM, laser scanning, photogrammetry, artificial intelligence.

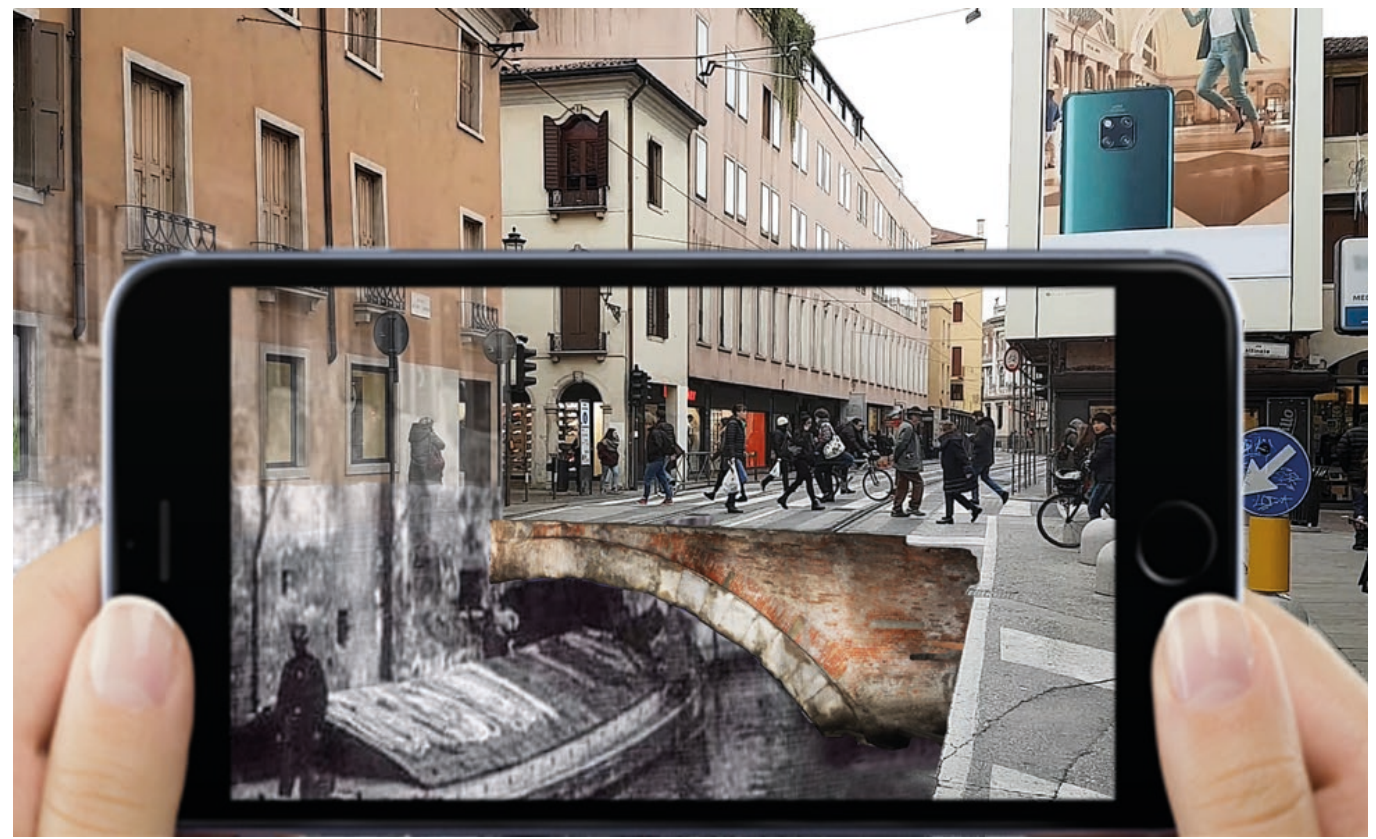




\section{Introduction}

PD-Invisible, an abbreviation that stands for 'PaDova INnovative VISions - visualizations and Imaginings Behind the city Learning', is a well rounded project that involved researchers from multiple fields. Archaeologists, building engineers, architects and informatic engineers collaborated for one year with the purpose to make visible what is, actually, invisible to the eyes of many. The project was funded by means of the European Social Fund and the Regione Veneto and included a partnership between the Department of Civil, Environmental and Architectural Engineering and the Department of Cultural Heritage of the University of Padua. The city has a beautiful yet, for the majority, invisible heritage belonging to the Roman age. The urban growth across the centuries and, sometimes, also the lack of attention and care, caused its disappearance. Thus the aim of the research was to promote and valorize these archaeological remains, bringing back their memory and, above all, trying to give them new life. Focus of the project was to create a digital environment suitable and available for both an expert and non-expert public, that provided the visualisation of the outcomes [Johnston et al. 2020; Perticarini, Marzocchella 202 I].

The chosen solution included the implementation of an application for smartphone that uses Augmented Reality (AR) and, moreover, Artificial Intelligence (AI). This could enable a simplified fruition of contents, diversifying them depending on the user's intentions and tastes. Multiple data, technical information about the structures, touristic paths and similar point of interests, historical narratives together with pictures and documents, are able to satisfy a wider public in the perspective of a cultural rediscovery of the Roman historical heritage of Padua. The research started with the identification of several points of interest across the city, structures connected by a common past and so linked with a touristic path. The Arena, near the Scrovegni Chapel, is the starting point as it is close to the actual train station, then walking south through the historical centre, along the Riviera dei Ponti Romani, there are Altinate and San Lorenzo Bridges, relevant Roman structures for trades and the economy of the city, hidden under the surface of the street with the tramline.

Finally, reaching Prato della Valle, we can find the foundation system of the Zairo Theatre, also hidden under the water of the canal of this huge and unique square [Bonetto, Pettenò, Veronese 2017]. San Lorenzo Bridge, between these structures, has an excellent conservation status and very positive conditions for the exploitation of the selected workflow. It is located under the street but enclosed in an underground chamber accessible with an old underpass built in the 1950's [Galliazzo 1941; Gasparotto 1951; Carraro et al. 2019].The division in phases of this research project included the initial data acquisition, the possibility to achieve an accurate 3D model with texture and information and the final $A R$ and $A l$ implementation.

\section{The Case Study}

As previously mentioned San Lorenzo Bridge is the case study identified as a starting point for developing research in all its phases and as a key to read for further in-depth analysis of the topic. The first very important operation that provides awareness and understanding of the chosen structure is data acquisition. The acquisition of the preliminar knowledge is then carried out, on the one hand through archival and documentary research to collect all the sources and testimonies regarding the bridge.

On the other hand, the survey performed using laser scanning and photogrammetry to obtain a virtual clone, geometrically similar. These models are the starting point for the subsequent modeling phase. Two different approaches were used: BIM modeling with the aim of providing a container for the information collected, allowing the exchange of data from different sources and the direct connection between different development environments, especially databases. The BIM model was chosen as the invisible backbone of data on which the realistic 3d model lean [Bonetto et al. 2019]. 


\section{Acquisition of the Invisible Heritages: Archival Memory and 3D Survey for the Digital Reconstruction}

Thanks to the permissions given by the following institutions was possible to carry out an extensive archival research: State Archives of Padua, San Gaetano - Altinate Cultural Center, Superintendence of Archeology, Fine Arts and Landscape, Archive of the University of Padua and Eremitani Civic Museum. It was possible to collect all the written and graphic documentation, regarding the main archaeological excavations and finds that during the centuries allowed to add important elements to the study of the Roman Age Padua. Alongside, the geometrical survey on-field of San Lorenzo Bridge covered the acquisition of two different types of survey, one with photogrammetry and the other with laser scanner technology. The Leica ScanStation P20, a time-of-flight scanner, was employed with the support of Leica HDS black/white target for the registration. A total of 18 scans were acquired, with resolution settings of 6,3 mm@ @ 0 m, and 78 targets were measured.The registration was performed by the software Leica Cyclone with a mean absolute error, checked on the targets, of $3 \mathrm{~mm}$. 24 vertices were measured as reference networks using a total station LeicaTCR I20 I and thanks to the GNSS was possible to obtain the geographic coordinates. Two photogrammetric surveys were also carried out: the first obtained by means of a Nicon D6 I 0 full frame reflex camera with a 35 $\mathrm{mm}$ lens; the second by means of two $12 \mathrm{mp}$ GoPro Hero 5 and focal length of $3 \mathrm{~mm}$. The photographs were taken following an orthogonal grid of $50 \times 50 \mathrm{~cm}$ for each arch of the bridge and the use of GoPro was useful in reaching the narrowest and most inaccessible areas. The point cloud generated by photogrammetry was compared with that obtained by laser scanning and no particular differences were found (fig. I).

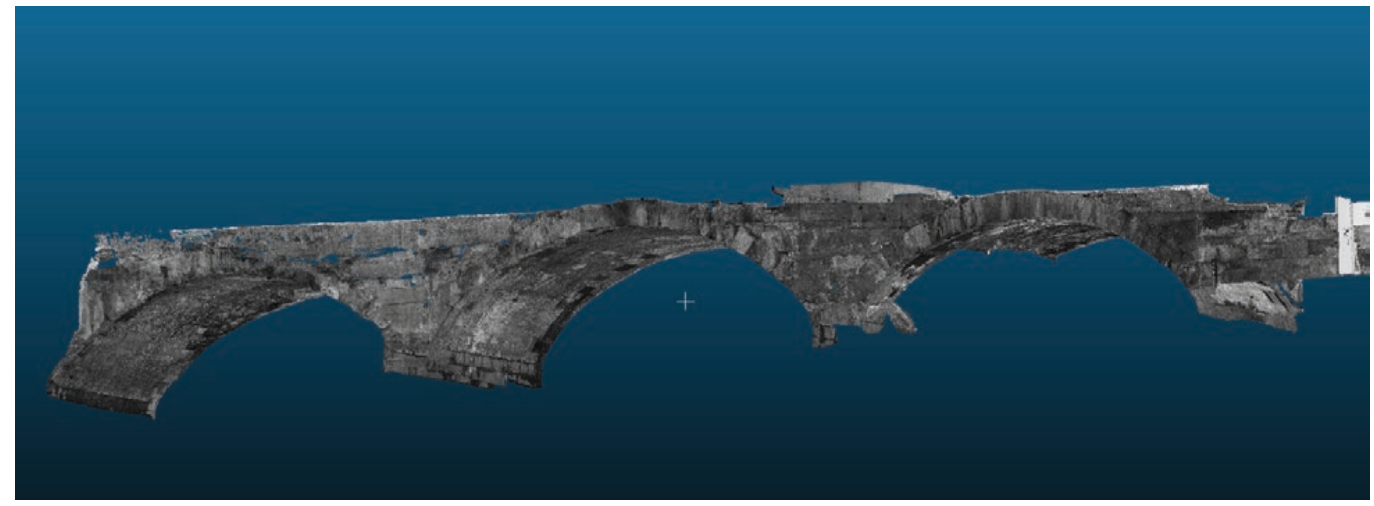

\section{D Modelling and Optimization for AR of the Photogrammetric Survey}

For the creation of the App, a precise workflow was identified to correctly display the 3D models on mobile devices. The first step consists in cleaning the point clouds and creating complex meshes with a high level of detail (the point cloud, the complex mesh and the related texture were obtained with the Agisoft Metashape software). The second phase consists in the optimization of the complex mesh by means of retopology (process that allows to drastically decrease the number of polygons of the mesh, making it lighter and more manageable on all smartphone models) [Palestini, Basso 2017] and by means of the baking of the normal map and diffuse map (process that allows to imprint the level of detail of the surfaces on the texture). For this purpose, the open source Blender 3D software was used for baking operations and the Instant Meshes tool for retopology operations [Perticarini et al. 2020]. Nowadays, with the latest software releases, two retopology systems have been implemented within the software: one based on the Quadriflow algorithm and one based on OpenVDB (Voxel). The third phase involves exporting the models to Unity software and developing the app in AR using Vuforia and Google's ARCore SDK. The App consists of a $\mathrm{UI}$ interface that allows navigation within the map and the identification of artifacts along the route; each artifact is visible both on site (through augmented reality) and remotely. It is 
Fig. 2. above, the complex mesh obtained from the point cloud; below, the simple mesh obtained from the retopology operation.
Fig. 3. Ul interface of the app. From left: the map, the interactive menus, the 3D model viewer, the glossary and the AR interface.

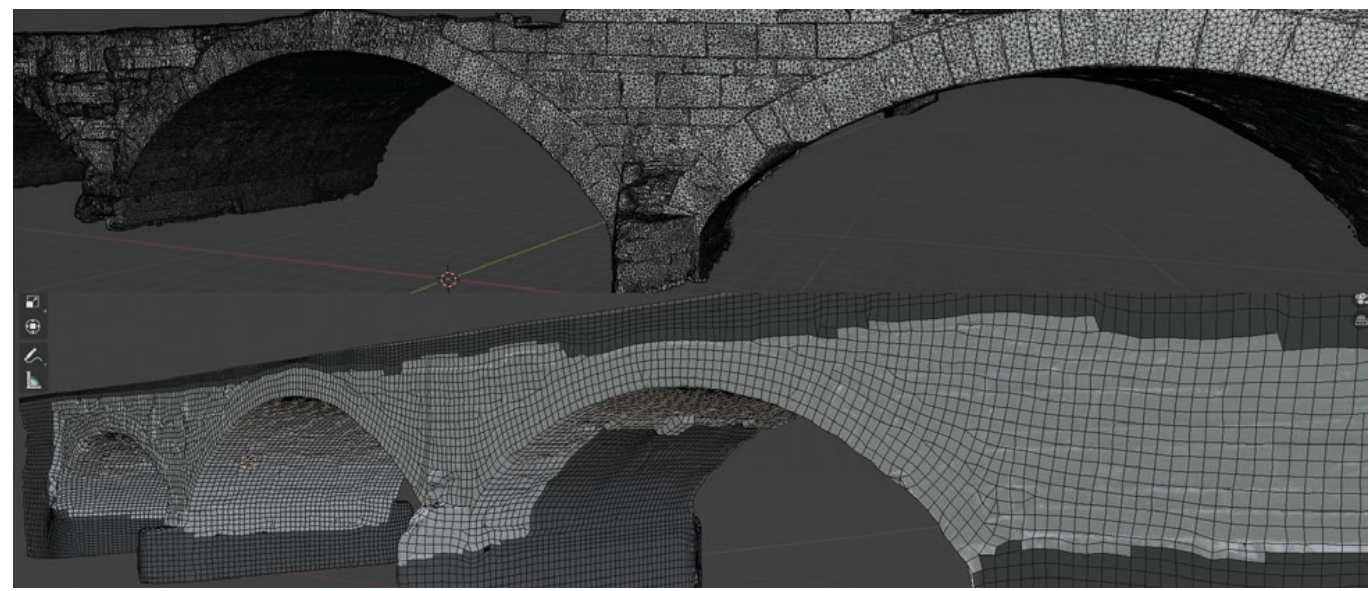

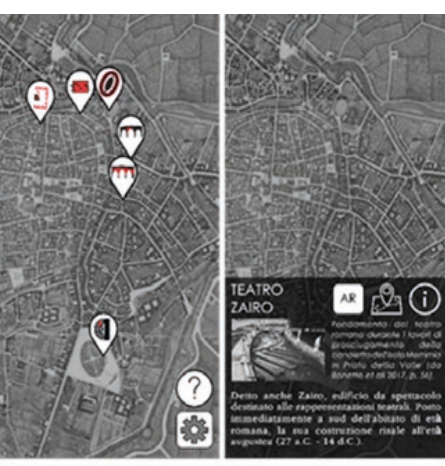

A

B

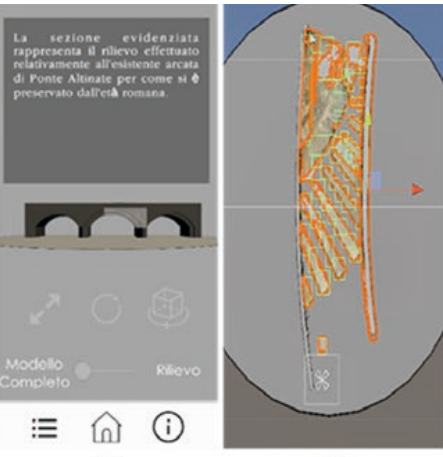

D

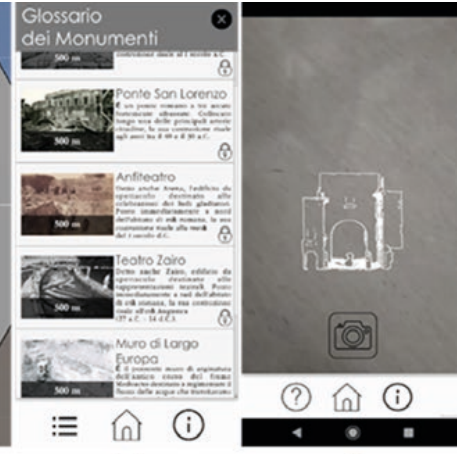

$\mathbf{F}$ also possible to view the historical information and the characteristics of the construction elements with a tap on the interactive $3 d$ model, thanks to the implementation of the BIM model on a layer below (figs. 2, 3).

\section{Future Implementations of Artificial Intelligence}

A further development of this type of applications could be the use of Al algorithms, in particular machine learning and artificial neural networks, which performs the function of satisfying the needs and interests of the user [Janković 2020]. An example could be to structure an algorithm capable of comparing the data coming from the GPS of the smartphone - relating to the most visited points of interest or the type of gait - with those coming from the camera (the photographs that the user takes most frequently or which artifacts are framed instead of others). Data mining consists in identifying information by extrapolating from large databases and allows, through associations and recurring patterns, to develop an ever-evolving App and an impulse for the user: thanks to the interpolation of data, it offers new suggestions based on the preliminary and continuous learning of the user's tastes [Ye, Qiu 202 I]. One of the most used data mining techniques is the neural networks (ANN or $\mathrm{NN}$ ) adaptive systems that change their structure based on external or internal information during the learning phases. The branch of information technology most suitable for the information coming from the camera is the automatic recognition and artificial vision [Stanisz et al. 202 I]. The computer vision system is composed of typical functions that can be differentiated into: image acquisition, pre-processing (resampling, noise reduction, contrast enhancement and space scaling), feature extraction (border lines or ridges ), detection or segmentation (small portions of images that are more useful for learning), processing and finally the decision-making process. As for image acquisition, current high-end smartphones are equipped with increasingly advanced sensors; in particular, the iPhone 12 Pro and Pro Max are equipped with a Lidar sensor, the portable version of the Lidar found in self-driv- 
ing cars for scanning and recognizing objects along the way [Shih, Diao, Chen 2019] [Yao 2020]. The sensor could be an excellent tool to improve the algorithm in the classification of artifacts framed by the smartphone. For the creation of APP of this type, PyTorch is used: a deep learning framework (class of machine learning algorithms) developed by Facebook Al Research (FAIR) group.This open source software allows you to facilitate the development of algorithms compatible with current Nvidia video cards and take advantage of the GPU.

\section{Conclusions}

Technological innovations are changing everyday objects into complex intelligent machines. The new sensors for acquiring the surrounding environment and the new processors make it possible to create projects that were unthinkable until a few years ago. it is obvious that often, the use of these technologies has negative sides, especially as regards the use of big data and privacy issues. With greater reason, innovations should be exploited to the maximum for more noble purposes, such as the enhancement and preservation of historical and cultural heritage.

\section{References}

Bonetto Jacopo, Giordano Andrea, Mazzariol Alessandro, Carraro Filippo and Callegaro Chiara (2019). PD-invisible : from drawing to city learning. In Belardi Paolo (ed.), Riflessioni : l'arte del disegno/ il disegno dell'arte. $41^{\circ}$ Convegno Internazionale dei Docenti delle Discipline della Rappresentazione. Roma: Gangemi.

Bonetto Jacopo, Pettenò Elena,Veronese Francesca (2017). Padova. La città di Tito Livio. Padova: Cleup.

Filippo Carraro, Monego Michele, Callegaro Chiara, Mazzariol Alessandro, Perticarini Maurizio, Menin Andrea, Achilli Vladimiro, Bonetto Jacopo, Giordano Andrea (2019). The 3d survey of the roman bridge of san lorenzo in padova (Italy): a comparison between sfm and tls methodologies applied to the arch structure. In International Archives of the Photogrammetry, Remote Sensing and Spatial Information Sciences, 42 (2MI5), pp. 255-262.

Galliazzo Vittorio ( 197I). I ponti di Padova Romana. Saggio di archeologia urbanistica. Padova: Cedam.

Gasparotto Cesira (195I). Padova romana. Roma: L'Erma di Bretschneider.

Janković Radmila (2020). Machine Learning Models for Cultural Heritage Image Classification: Comparison Based on Attribute Selection. In Information, I I ( I), pp. I- I3.

Lesley Johnston, Galloway Romy, Trench Jordan John, Poyade Matthieu, Tromp Jolanda, My Hoang Thi (2020). Augmented Reality at Heritage Sites. In Tromp Jolanda G., Le Dac-Nhuong, Le Chung Van (eds.). Emerging Extended Reality Technologies For Industry 4.0: Early Experiences with Conception, Design, Implementation, Evaluation and Deployment. Hoboken:Wiley, pp. I0 I - 19.

Palestini Caterina, Basso Alessandro (2017). The Photogrammetric Survey Methodologies Applied to Low Cost 3D Virtual Exploration in Multidisciplinary Field. In International Archives of the Photogrammetry, Remote Sensing and Spatial Information Sciences, 42 (2W8), pp. 195-202.

Perticarini Maurizio, Callegaro Chiara, Carraro Filippo, Mazzariol Alessandro (2020). Two Methods of Optimization for an Ar Project: Mesh Retopology and Use of Pbr Materials. In Cicalò Enrico (ed.). Advances in Intelligent Systems and Computing, vol | | 40. Springer: Cham.

Perticarini Maurizio, Valeria Marzocchella (2021). 3D Animation Applied to Street Art. Physical and Remote Connections Aimed at Inclusion. In Cheng Liang-Yee (ed.). ICGG 2020 - Proceedings of the 19th International Conference on Geometry and Graphics, pp. 920-923.

Shih Naai Jung, Pei Huang Diao, Yi Chen (2019). ARTS, an AR Tourism System, for the Integration of 3D Scanning and Smartphone AR in Cultural Heritage Tourism and Pedagogy. In Sensors, 19 (17), 3725.

Stanisz Joanna, Konrad Lis, Tomasz Kryjak, Marek Gorgon (2021). Hardware-Software Implementation of the PointPillars Network for 3D Object Detection in Point Clouds. In Workshop on Design and Architectures for Signal and Image Processing ( I th Edition), DASIP '2 I. New York, NY, USA: Association for Computing Machinery, pp. 44-5 I.

Yao Zhennan (2020). VTQuestAR : An Augmented Reality Mobile Software Application for Virginia Tech Campus Visitors. Virginia Tech.

Ye Yang, Hongfei Qiu (202I). Exploring Affecting Factors of Park Use Based on Multisource Big Data: Case Study in Wuhan, China. In Journal of Urban Planning and Development, I 47 ( I), 05020037, pp. I - I I.

\section{Authors}

Maurizio Perticarini, Dept. of Architecture and Industrial Design, University of Campania LuigiVanvitelli, maurizio.perticarini@unicampania.it Chiara Callegaro, Dept. of Civil, Environmental and Architectural Engineering, University of Padua, chiara.callegaro@unipd.it 
